Case Series

\title{
Management of benign paediatric thyroid nodules: experience from paediatric ENT services of a tertiary care hospital
}

\author{
Arunabha Chakravarti*, Sneha Chandrasekhar
}

Department of ENT, Lady Hardinge Medical college and Kalawati Saran Children's Hospital, New Delhi, India

Received: 17 February 2021

Accepted: 18 March 2021

\section{*Correspondence:}

Dr. Arunabha Chakravarti,

E-mail: drchakravarti@yahoo.co.in

Copyright: (c) the author(s), publisher and licensee Medip Academy. This is an open-access article distributed under the terms of the Creative Commons Attribution Non-Commercial License, which permits unrestricted non-commercial use, distribution, and reproduction in any medium, provided the original work is properly cited.

\begin{abstract}
Thyroid nodules are a common occurrence in adults but are rarely seen in children. We present here four cases of paediatric thyroid nodules. Patients were subjected to a thorough clinical evaluation, blood thyroid level testing, ultrasonography (USG) and fine needle aspiration cytology (FNAC). The size of the nodules ranged from $2 \mathrm{~cm}$ to 7.5 $\mathrm{cm}$. All four nodules were reported as "benign" on FNAC, of which three under-went total thyroidectomy owing to the bi-laterality and large size of the nodules. None of the cases had post-operative complications of hypocalcemia or recurrent laryngeal nerve paralysis. Of the 3 patients taken up for surgery one patient was found to have papillary thyroid carcinoma. Subsequent radiological investigation showed no residual disease. All three operated cases were followed-up in the ENT outpatient department for at least 2 years and showed no signs of recurrence. One patient with a small thyroid nodule $(2 \mathrm{~cm})$ was managed non - surgically and has been kept on follow up since 12 months. In our experience, total thyroidectomy, even in a benign disease, in expert hands, is a safe procedure and the best management option in children with large goiter, to avoid recurrence and thereby a redo surgery.
\end{abstract}

Keywords: Thyroid nodule, Children, Paediatric thyroidectomy, Total-thyroidectomy

\section{INTRODUCTION}

Thyroid nodules are uncommon in the paediatric age group, affecting only $1-2 \%$ of its population. ${ }^{1}$ Similar to adults, the majority of paediatric thyroid nodules are benign. However, there is a higher risk of malignancy in children (22-26\%) as compared to adults (5\%), thereby warranting a more aggressive approach towards its timely diagnosis and management. ${ }^{2}$ Most children with both benign and malignant nodules present as asymptomatic neck masses. A thorough clinical evaluation is required along with diagnostic tests including thyroid function tests, radiological and cytological assessment. Thyroid surgery has been the mainstay in management of paediatric thyroid nodules. However, the extent of surgery remains controversial. Currently, the preferred surgical options are lobectomy with isthmectomy for benign thyroid nodules and total thyroidectomy for malignant or nodules suspicious of malignancy. ${ }^{2}$ Fine needle aspiration cytology (FNAC) and ultrasonography (USG) facilitate in determining a nodule to be benign or malignant. However, there is insufficient data in children on the rate of false negative "benign" pathology diagnosed based on FNAC and USG features. ${ }^{2}$ The risks and benefits of surgery along with proficiency of the surgeon must be considered while planning the management. With this background, we present a case series of four paediatric thyroid nodules, highlighting the management of such patients.

\section{CASE SERIES}

\section{Case 1}

A 11-year-old female presented with history of swelling in front of her neck since past 5 years, which was gradually increasing in size with no other compressive or associated symptoms. On examination, two swellings 
were seen in anterior part of neck, measuring around $3 \times 2 \mathrm{~cm}$ on right side and $5 \times 3 \mathrm{~cm}$ on left side (Figure 1). It was firm in consistency, ton-tender, had a smooth surface and moved upwards with deglutition. Systemic examination was unremarkable. Ultrasound of neck revealed a multinodular goiter. FNAC was suggestive of colloid goiter with cystic changes (Bethesda category 2).

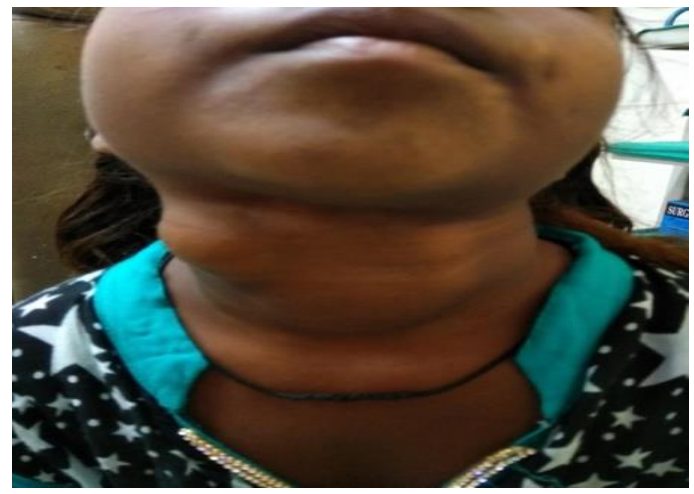

Figure 1: 11-year old female with thyroid nodule.

Thyroid function test was within normal limits. Patient was taken up total thyroidectomy under general anesthesia. Intra-operatively both lobes of thyroid were found to be involved and removed while preserving bilateral recurrent laryngeal nerve and parathyroid glands. Post-operative period was uneventful and final histopathological report was consistent with colloid goiter. Following a paediatric endocrinology consultation, patient was started on $50 \mathrm{mcg}$ of tablet levothyroxine. Patient was having been on follow up in E.N.T outpatient department for last 4 years and has no recurrence.

\section{Case 2}

A 10-year-old male child presented with complains of anterior neck swelling which was present since birth. It was allegedly small and approximately $<1 \mathrm{~cm}$ in size initially but rapidly increased in size over last 6 months. On examination, a solitary ovoid swelling of size $7.5 \times 6.5$ $\mathrm{cm}$, with firm consistency was found covering the entire anterior part of neck. The swelling extended up to $3 \mathrm{~cm}$ below the chin superiorly, $2 \mathrm{~cm}$ above suprasternal notch inferiorly and laterally up to the anterior border of bilateral sternocleidomastoid mastoid muscle (Figure 2). There was restricted movement of the swelling with deglutition and no palpable lymph nodes. Ultrasonography showed Multinodular goiter involving both lobes of thyroid gland with slightly increased vascularity and no lymphadenopathy. Blood thyroid profile was normal. FNAC was suggestive of colloid goitre. Pre-operatively flexible fiberoptic laryngoscopy was done and bilateral vocal cords were found to be mobile. The patient underwent total thyroidectomy and post-operative histopathology showed papillary carcinoma of thyroid. Subsequently, radio iodine scan showed no uptake and thyroglobulin level was $0.2 \mathrm{ng} / \mathrm{ml}$.
After a paediatric endocrinology consultation, patient was started on levothyroxine replacement. Patient is being followed up for last 3 years and has not developed recurrence.

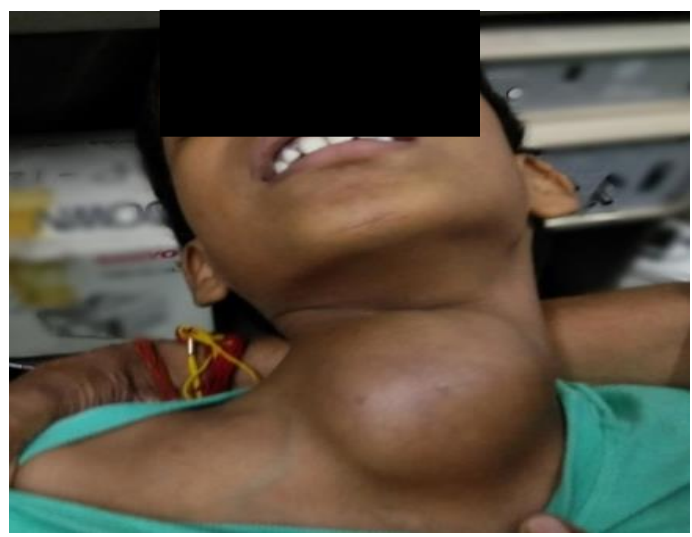

Figure 2: 10-year old male.

Case 3

A 3-year-old male presented with swelling on right side neck for since 6 months of age. There was no history of rapid increase in size of the swelling or any other symptoms. On examination a solitary nodule of size $2 \times 2$ $\mathrm{cm}$ was seen on right side of neck (Figure 3). USG neck showed colloid nodule involving the right lobe of thyroid gland and USG guided FNAC was suggestive of colloid goiter. Blood thyroid profile was within normal limit. Due to small size of the nodule and benign USG and FNAC features, patient has been kept on a regular follow up in the ENT OPD with biannual USG to monitor the nodule.

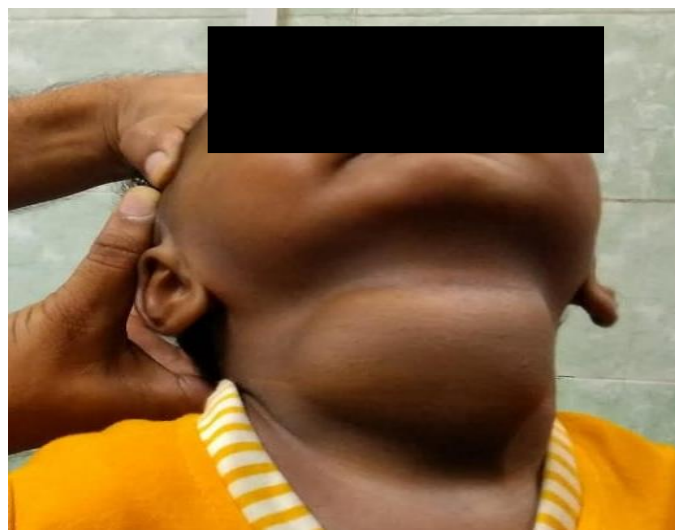

Figure 3: 3-year-old male.

\section{Case 4}

A 3-year-old male child was brought to the ENT OPD with a neck swelling present since birth and gradually increasing in size. Parents also mentioned complaints of discomfort on swallowing food since last 6 months. On examination, a diffuse swelling of size $6 \times 6 \mathrm{~cm}$ was seen in the anterior part of neck (Figure 4) with no palpable 
neck lymph nodes. Patient was clinically euthyroid and thyroid function test was normal. USG neck revealed multinodular goiter involving both lobes of thyroid with cystic changes. FNAC was suggestive of colloid goiter. Patient underwent total thyroidectomy. Intra-operatively both lobes of thyroid gland were found to be involved and removed, preserving both recurrent laryngeal nerves and parathyroid glands. Post - operative histopathology was consistent was pre-operative cytology. Child was started on daily $25 \mathrm{mcg}$ of levothyroxine and is being followed up monthly in paediatric endocrinology clinic. Patient is being followed up clinically for last 2 years and has shown no recurrence.

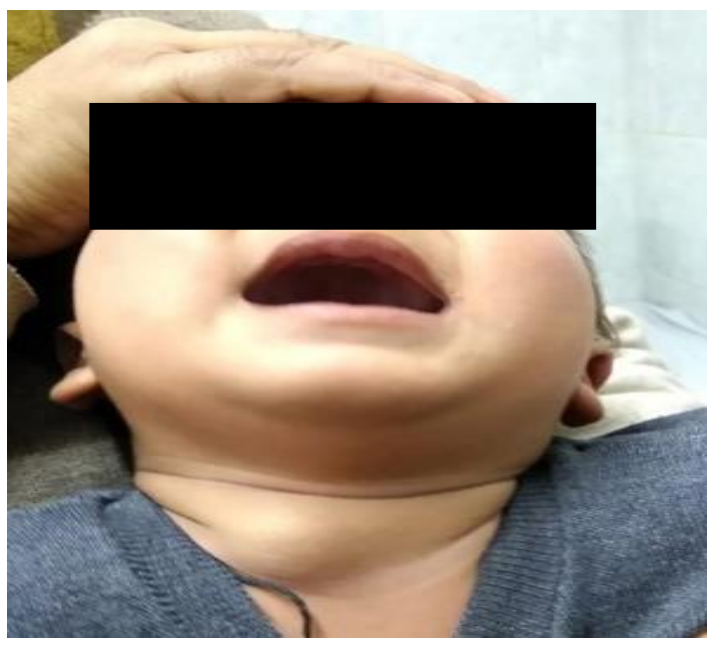

Figure 4: 3-year-old male.

\section{DISCUSSION}

Thyroid nodules in paediatric population is a rare entity but over last several years there has been an increase in its incidence. ${ }^{3}$ Thyroid nodular disease can be solitary nodule, multinodular goitre, nodular goitre seen in autoimmune thyroid disease (Hashimoto's/grave's disease and non - palpable thyroid nodule. ${ }^{4}$ Although palpable thyroid nodules are less common in children than adults but sonographic and or pathological abnormalities are common $\left(0.2-5 \%\right.$ of children, $13 \%$ of adolescents). ${ }^{5}$ Children with thyroid nodules have a reportedly higher incidence of malignancy and more likely to present with regional metastasis and extrathyroidal extension. ${ }^{2}$ Differentiating malignant from benign lesion is a challenging dilemma in this population. ${ }^{6}$

There are established guidelines in management of thyroid nodules in adult, however, its. evaluation and management in children remains controversial. The latest American thyroid association (ATA) guidelines recommends thyroid and neck ultrasound in all children with a suspected or incidental thyroid nodule. ${ }^{2}$ In adults, FNAC is not necessary in patients with nodules $<1 \mathrm{~cm}$ with no previous history of radiation exposure or pathological regional lymph node. However, in children ultrasound characteristics and clinical context are used to identify thyroid nodules that warrant FNAC. ${ }^{2}$ Ultrasound features such as hypo-echogenicity, irregular margins, and increased intra-nodular blood flow, microcalcifications and abnormal cervical lymph-node point towards increased risk of malignancy. ${ }^{2}$ USG guided FNAC is recommended in children due to higher proportion of malignancy and the potential difficulty in obtaining repeat samples in children. ${ }^{2}$

The surgical approach is dictated by FNAC results. ${ }^{2}$ Whenever surgery is required, the surgical options and other concerns associated with the procedure must be discussed between the surgeon and the parents / guardians of the child. As per ATA recommendation, those with benign cytology and no high - risk ultrasound features can be kept on an ultrasound follow-up. Abiding by this, owing to the small size and benign pathology of the of nodule in case 3 of our series, the patient is being followed up by serial ultrasound. For growing nodules or those with suspicious ultrasound features, repeat FNAC should be performed to assist with surgical planning. ${ }^{2}$

Surgical intervention is recommended for nodules causing compressive symptoms, nodules $>4 \mathrm{~cm}$ due to high false - negative rates in larger lesions, for cosmesis, or by patient/parent's choice. ${ }^{2}$ ATA recommends lobectomy with or without isthmectomy for nodules that are benign on FNAC with no high - risk ultrasound feature. In cases of bilateral nodularity when cancer is clinically suspected and cytologically confirmed; total thyroidectomy is recommended. Case 1, 2 and 4 from our series, with benign FNA cytology underwent total thyroidectomy. Total thyroidectomy was favoured over lobectomy \pm isthmectomy in these cases due to the large size of the nodules $(>4 \mathrm{~cm})$, involvement of bilateral lobes and compressive symptoms (case 4). In 2 out of the 3 above mentioned cases the post-operative histopathology report was consistent with the respective pre-surgery FNA results. However, case 2 in this series, although had a being cytology on FNA, the postoperative histopathological examination revealed papillary carcinoma. Large thyroid nodules are known to have an increased chance of sampling error and a higher false negative which is reinforced by the aforementioned case. $^{2}$ In our opinion, it would be wise to manage large thyroid nodules in children with total/near total thyroidectomy. It precludes the need for an additional surgical procedure in event of a malignant lesion discovered in post - operative histopathology. While molecular testing has shown to aid in diagnosis of indeterminate nodules in adults, it has not yet been validated in children. ${ }^{2}$ At present, there is scarce literature on the role of genetic testing to unfailingly rule out malignancy in children. ${ }^{2}$

Recently, there has been an increasing support for total thyroidectomy as a first line management option in benign thyroid disease. $^{7}$ It not only provides histopathological specimen for final tissue diagnosis, but also has lower rate of recurrence. ${ }^{8}$ This is of utmost 
importance, since there is a two to three-fold higher risk of malignancy in paediatric thyroid nodules. ${ }^{2}$ Limiting factors of total thyroidectomy in children include the need for life long thyroid supplementation, risk of damage to parathyroid glands and recurrent laryngeal nerve. Recurrent laryngeal nerve (RLN) injury is a devastating complication associated with thyroid surgery but the incidence of RLN injury in total thyroidectomy is similar to that of lobectomy. ${ }^{8}$ Complications associated with total thyroidectomy, including hypocalcaemia and RLN injury was not observed in any patient in our case series. Similar to that of adults, patient outcomes in paediatric cases also appear to be associated with surgeon volume. ${ }^{9}$ Total-thyroidectomy, while not without risks, is a safe procedure for management of benign paediatric thyroid nodules, at the hands of an experienced surgeon.

\section{CONCLUSION}

Management of paediatric thyroid disease has been a controversial topic due to its relative rarity. At present, there is no universal consensus at present on the extent of surgical excision for treatment of benign nodules, with many procedures undertaken secondary to surgeon's preference and expertise. While lobectomy +/isthumectomy is the preferred procedure for benign nodules, one must consider total thyroidectomy in children with large thyroid nodules, keeping in mind the higher incidence of malignancy in paediatric thyroid nodule. FNAC is an important diagnostic tool to differentiate benign and malignant lesion but not fully reliable in large lesions $(>4 \mathrm{~cm})$. There is scope for future research based on molecular testing in determining malignant potential of such nodules and on establishing management protocol for paediatric thyroid nodules.

Funding: No funding sources Conflict of interest: None declared

Ethical approval: Not required

\section{REFERENCES}

1. Burch HB. Evaluation and management of the solid thyroid nodule. Endocrino Metabol Clinics North Am. 1995;24(4):663-710.

2. Francis GL, Waguespack SG, Bauer AJ, Angelos P, Benvenga S, Cerutti JM, et al. Management guidelines for children with thyroid nodules and differentiated thyroid cancer: the American Thyroid Association guidelines task force on pediatric thyroid cancer. Thyroid. 2015;25(7):716-59.

3. Hogan AR, Zhuge Y, Perez EA, Koniaris LG, Lew JI, Sola JE. Pediatric thyroid carcinoma: incidence and outcomes in 1753 patients. J Surgic Res. 2009;156(1):167-72.

4. Niedziela M. Pathogenesis, diagnosis and management of thyroid nodules in children. Endoc Canc. 2006;13(2):427-53.

5. Mussa A, De Andrea M, Motta M, et al. Predictors of Malignancy in Children with Thyroid Nodules. J Pediatr. 2015;167:886-92.

6. Hung W. Solitary thyroid nodules in 93 children and adolescents: A 35-years experience. Horm Res. 1999;52:15-8.

7. Raval MV, Browne M, Chin AC, Zimmerman D, Angelos P, Reynolds M. Total thyroidectomy for benign disease in the pediatric patient-feasible and safe. J Pediat Surg. 2009;44(8):1529-33.

8. Barczynski M, Konturek A, Stopa M, Cichon S, Richter P, Nowak W. Total thyroidectomy for benign thyroid disease: is it really worthwhile?. Ann Surg. 2011;254(5):724-30.

9. Sosa JA, Bowman HM, Tielsch JM, Powe NR, Gordon TA, Udelsman R. The importance of surgeon experience for clinical and economic outcomes from thyroidectomy. Ann Surg. 1998;228(3):320.

Cite this article as: Chakravarti A, Chandrasekhar S Management of benign paediatric thyroid nodules: experience from paediatric ENT services of a tertiary care hospital. Int J Otorhinolaryngol Head Neck Surg 2021;7:672-5. 